\title{
Influência da anquiloglossia neonatal no aleitamento materno: revisão de literatura
}

Influence of neonatal ankyloglossia on breastfeeding: a literature review

Influencia de la anquiloglosia neonatal en lactancia materna: una revisión de la literatura

Ytalo Lourenço MACIEL ${ }^{1}$

Adriano Referino da SILVA SOBRINHO

Juliana de Godoy Bezerra MEDRADO²

${ }^{1}$ Curso de Bacharelado em Odontologia da Universidade de Pernambuco campus Arcoverde, 56503-146 Arcoverde-PE, Brasil

${ }^{2}$ Professora Titular, Faculdade de Odontologia do Recife (FOR) Fundação Odontologica Presidente Castello Branco- FOPCB 50100-280 Recife - PE, Brasil

\section{Resumo}

Introdução: Anomalia de desenvolvimento lingual, a anquiloglossia é caracterizada pela inserção atípica do frênulo lingual. Tal condição causa um impedimento na correta movimentação da língua e prejuízos a diversas áreas do sistema estomatognático. Objetivo: Realizar uma busca na literatura acerca das características da anquiloglossia neonatal e suas repercussões no sistema estomatognático que influenciam no aleitamento materno. Material e Método: Foram utilizados artigos disponíveis nas bases de dados PubMed e Biblioteca Virtual em Saúde (BVS), publicados nos idiomas inglês e português e publicados entre os anos de 2009 a 2019. Resultados: Após a aplicação dos critérios de inclusão e exclusão, foram selecionados 11 estudos. A anquiloglossia é uma é bastante estudada pela literatura, porém sua relação com o aleitamento materno ainda causa controvérsias entre alguns autores. Conclusão: A literatura aponta a influência sobre o freio lingual e sua implicação na nutrição nutritiva do lactente. Porém, mais parâmetros e estudos longitudinais para obter dados concisos acerca do correto diagnóstico e momento de tratamento desses bebês, ainda são necessários.

Descritores: Anquiloglossia; Freio Lingual; Odontopediatria.

\section{Abstract}

Introduction: Ankyloglossia is a developmental anomaly of the tongue characterized by the atypical insertion of the lingual frenulum. Such condition causes an impediment in the correct movement of the tongue and damages to several areas of the stomatognathic system. Objective: To conduct a literature search on the characteristics of neonatal ankyloglossia and its repercussions on the stomatognathic system that influence breastfeeding. Material and Method: Articles available in the PubMed and Virtual Health Library (VHL) databases, published in English and Portuguese and published between 2009 and 2019, were used. Results: After applying the inclusion and exclusion criteria, 11 studies were selected. Ankyloglossia is one that has been extensively studied in the literature, but its relationship with breastfeeding is still controversial among some authors. Conclusion: The literature points to the influence on the tongue brake and its implication in the infant's nutritional nutrition. However, more parameters and longitudinal studies to obtain concise data about the correct diagnosis and timing of treatment of these babies are still needed.

Descriptors: Ankyloglossia; Lingual Frenum; Pediatric Dentistry.

\section{Resumen}

Introducción: La anquiloglosia es una anomalía del desarrollo lingual caracterizada por la inserción atípica del frenillo lingual. Tal condición causa un impedimento en el movimiento correcto de la lengua y daña varias áreas del sistema estomatognático. Objetivo: Realizar una búsqueda bibliográfica sobre las características de la anquiloglosia neonatal y sus repercusiones en el sistema estomatognático que influyen en la lactancia materna. Material y Método: Se utilizaron los artículos disponibles en las bases de datos de PubMed y Virtual Health Library (VHL), publicados en inglés y portugués y publicados entre 2009 y 2019. Resultados: Después de aplicar los criterios de inclusión y exclusión, seleccionamos 11 estudios. La anquiloglosia es ampliamente estudiada en la literatura, pero su relación con la lactancia materna todavía causa controversia entre algunos autores. Conclusión: La literatura señala la influencia en el freno de lengua y su implicación en la nutrición nutricional del lactante. Sin embargo, aún se necesitan más parámetros y estudios longitudinales para obtener datos concisos sobre el diagnóstico correcto y el momento del tratamiento de estos bebés.

Descriptores: Anquiloglosia; Frenillo Lingual; Odontología Pediátrica.

INTRODUÇÃO

O aleitamento materno está intrinsecamente ligado à sucção e deglutição, que devem funcionar de forma coordenada com a respiração ${ }^{1}$. A movimentação da língua exerce um papel fundamental nesse processo de sucção nutritiva do neonato, e qualquer restrição à livre movimentação da língua pode comprometer as funções de ganho de peso e desenvolvimento por conta de um desmame prematuro ${ }^{2}$.

O começo do desenvolvimento da língua se dá na quarta semana de vida intrauterina. Em seguida é formada a base da língua, que quando chega a seu estágio final, torna-se uma fina faixa de tecido, formando o freio lingual. Durante essa fase podem ocorrer distúrbios que resultam na anteriorização da inserção do freio lingual, deixando-o mais largo ou encurtado gerando a anquiloglossia ${ }^{3}$.
A anquiloglossia é uma anomalia de desenvolvimento lingual caracterizada pela inserção baixa do frênulo lingual ou a inserção aumentada do músculo genioglosso ${ }^{4}$. A etiopatogenia da anquiloglossia ainda não é bem conhecida, mas existem estudos que apontam que a "língua presa" possa estar associada a mutações genéticas ou alterações congênitas como a fenda palatina e hipodontia ${ }^{5,6}$. Existem características indicativas da anquiloglossia quanto a anatomia do freio lingual, como o freio anormalmente curto e inserido próximo ao ápice da língua; dificuldade nos movimentos de elevação, lateralidade e protrusão linguais; e quando a língua assume um aspecto de coração quando em protrusão ${ }^{7}$.

O diagnóstico de anquiloglossia deve ser feito precocemente com o intuito de diminuir os danos à saúde do lactente, otimizando o ganho 
de peso e garantindo a mãe um conforto maior durante a amamentação ${ }^{1}$. Diante dessa perspectiva, em 2014, foi aprovada uma lei que obriga do diagnóstico precoce de alterações que acometem o frênulo lingual do neonatal em todos os hospitais e a maternidades vigentes em território nacional ${ }^{8}$. Há um protocolo de avaliação do frênulo lingual em bebês, que objetiva 0 diagnóstico das alterações características da anquiloglossia, indicando ou não, a realização de frenotomia lingual ${ }^{2,9}$.

Entretanto, mesmo com uma legislação vigente que assegure ao neonatal e a puérpera um serviço de diagnóstico ainda na maternidade ou hospital, há a escassez de recursos para a realização dessa nova adequação. Cabe ao pediatra ou profissional de saúde que estejam acompanhando 0 bebe na primeira consulta deverão encaminha-lo para um local de referência para este exame $e^{7,10}$.

Diante do cenário exposto, o presente estudo tem como objetivo revisar a literatura acerca das características da anquiloglossia neonatal e suas repercussões no sistema estomatognático que influenciam no aleitamento materno.

\section{MATERIAL E MÉTODO}

Trata-se de uma revisão de literatura do tipo narrativa, a qual descreve um assunto específico a partir da análise da literatura, da interpretação e do julgamento crítico pessoal do pesquisador ${ }^{11}$. Esse tipo de trabalho é fundamental na educação continuada dos pesquisadores pois possibilita a aquisição e atualização de conhecimento sobre um determinado tema em curto período de tempo ${ }^{12}$. A estratégia de busca dos estudos se deu através das bases de dados, do PubMed e Biblioteca Virtual em Saúde (BVS) durante o período de meses de abril a maio de 2019, utilizando-se dos descritores "Anquiloglossia", "Recém-nascido" e "Freio Lingual". Como critérios de inclusão foram selecionados estudos com textos completos, publicados nos idiomas inglês e português.

A análise dos estudos ocorreu em duas etapas. Na primeira, após o cruzamento dos descritores, foi realizada a leitura dos títulos e resumos dos artigos encontrados. Na segunda etapa, os estudos pré-selecionados foram analisados na íntegra, e aqueles que não se encaixaram nos critérios de inclusão foram dispensados. Os critérios de inclusão foram: estudos publicados na íntegra, nos idiomas inglês ou português; que abordaram 0 diagnóstico e avaliação do freio lingual em bebês e impacto a curto médio e longo prazo.
Como critério de exclusão optou-se por não incluir os artigos publicados antes do ano de 2009.

\section{RESULTADOS}

A estratégia de busca resultou em onze estudos selecionados cujos principais aspectos são descritos e sintetizados na Tabela 1.

\begin{tabular}{|c|c|c|c|}
\hline Estudo & Título do estudo & Objetivos do estudo & Amostra \\
\hline $\begin{array}{l}\text { Morisso et } \\
\text { al. } .^{13}(2012)\end{array}$ & $\begin{array}{l}\text { Ankyloglossiarelated } \\
\text { changes in the } \\
\text { stomatognathic system. }\end{array}$ & $\begin{array}{lr}\text { Verificar a ocorrência de } \\
\text { alterações do } & \text { sistema } \\
\text { estomatognático } & \mathrm{em} \\
\text { pacientes } & \text { com } \\
\text { anquiloglossia. } & \end{array}$ & $\begin{array}{l}1.516 \\
\text { pacientes, } \\
\text { entre } 5 \text { e } 16 \\
\text { anos }\end{array}$ \\
\hline $\begin{array}{l}\text { Pransly et } \\
\text { al.14 (2015) }\end{array}$ & $\begin{array}{l}\text { Breastfeeding } \\
\text { difficulties and oral } \\
\text { cavity anomalies: The } \\
\text { influence of posterior } \\
\text { ankyloglossia and } \\
\text { upper-lipties. }\end{array}$ & $\begin{array}{l}\text { Descrever a nossa } \\
\text { experiência em } \text { uma } \\
\text { clínica de dificuldade de } \\
\text { amamentação de alto } \\
\text { volume com foco } \\
\text { na anquiloglossia posterio } \\
\text { r. }\end{array}$ & 618 pacientes \\
\hline $\begin{array}{l}\text { Wakhanritt } \\
\text { ee et al.15 } \\
\text { (2016) }\end{array}$ & $\begin{array}{l}\text { The outcomes of a } \\
\text { frenulotomy on } \\
\text { breastfeeding infants } \\
\text { followed up for } 3 \\
\text { months at Thammasat } \\
\text { University Hospital. }\end{array}$ & $\begin{array}{l}\text { Estudar os efeitos da } \\
\text { frenotomia na dor } \\
\text { mamilar, travamento e } \\
\text { sucesso no aleitamento } \\
\text { materno exclusivo aos } 3 \\
\text { meses de seguimento. }\end{array}$ & $\begin{array}{l}\text { 328-mães de } \\
\text { infantes }\end{array}$ \\
\hline $\begin{array}{l}\text { Fujinaga et } \\
\text { al. }^{16}(2017)\end{array}$ & $\begin{array}{l}\text { Frênulo lingual e } \\
\text { aleitamento materno: } \\
\text { estudo descritivo. }\end{array}$ & $\begin{array}{l}\text { Avaliar o frênulo da língua } \\
\text { em bebês recém-nascidos } \\
\text { a termo e verificar sua } \\
\text { associação com } \\
\text { aleitamento materno. }\end{array}$ & $\begin{array}{l}139 \text { binômios } \\
\text { mãe/bebê }\end{array}$ \\
\hline $\begin{array}{l}\text { Muldoon et } \\
\text { al. }{ }^{17}(2017)\end{array}$ & $\begin{array}{l}\text { Effect of frenotomy on } \\
\text { breastfeeding variables } \\
\text { in infants with } \\
\text { ankyloglossia (tongue- } \\
\text { tie) a prospective } \\
\text { before and after cohort } \\
\text { study. }\end{array}$ & $\begin{array}{lr}\text { Determinar os efeitos } \\
\text { associados à frenotomia } \\
\text { nas variáveis r da } \\
\text { amamentação } & \text { em } \\
\text { lactentes } & \\
\text { com anquiloglossia. }\end{array}$ & 98 mães \\
\hline $\begin{array}{l}\text { Dixon et al. } \\
{ }^{18}(2018)\end{array}$ & $\begin{array}{l}\text { A multifaceted } \\
\text { programme to reduce } \\
\text { the rate of tongue-tie } \\
\text { release surgery in } \\
\text { newborn infants: } \\
\text { Observational study. }\end{array}$ & $\begin{array}{l}\text { Avaliar o diagnóstico e o } \\
\text { tratamento da liberação de } \\
\text { frênulo em recém- } \\
\text { nascidos com suspeita de } \\
\text { amarração na língua } \\
\text { (anquiloglossia), apoiando } \\
\text { a amamentação e } \\
\text { garantindo que cirurgias } \\
\text { desnecessárias fossem } \\
\text { evitadas. }\end{array}$ & 309 bebês \\
\hline $\begin{array}{l}\text { Martinelli19 } \\
(2018)\end{array}$ & 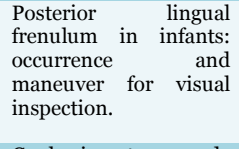 & $\begin{array}{l}\text { Verificar a ocorrência do } \\
\text { frênulo lingual posterior } \\
\text { ou submucoso, bem como, } \\
\text { avaliar a eficiência de uma } \\
\text { manobra que possibilita } \\
\text { sua visualização. }\end{array}$ & 1715 lactantes \\
\hline $\begin{array}{l}\text { omini et al. } \\
{ }_{20}(2018)\end{array}$ & $\begin{array}{l}\text { Conhecimento de } \\
\text { gestantes sobre o teste } \\
\text { da linguinha em } \\
\text { neonates. }\end{array}$ & $\begin{array}{l}\text { Verificar o conhecimento } \\
\text { sobre teste da linguinha e } \\
\text { sua relação com o perfil } \\
\text { sociodemográfico das } \\
\text { gestantes. }\end{array}$ & 427 gestantes \\
\hline $\begin{array}{l}\text { Araújo et } \\
\text { al. }{ }^{21}(2019)\end{array}$ & $\begin{array}{l}\text { Evaluation of the } \\
\text { lingual frenulum in } \\
\text { newborns using two } \\
\text { protocols and its } \\
\text { association } \\
\text { breastfeeding. }\end{array}$ & $\begin{array}{l}\text { Caracterizar o frênulo } \\
\text { lingual de recém-nascidos } \\
\text { utilizando } \\
\text { dois protocolos diferentes. }\end{array}$ & $\begin{array}{l}449 \\
\text { mães/bebês }\end{array}$ \\
\hline $\begin{array}{l}\text { Campanha } \\
\text { et al. } 22 \\
\text { (2019) }\end{array}$ & $\begin{array}{lr}\text { Associação } & \text { entre } \\
\text { anquiloglossia } & \text { e } \\
\text { amamentação. } & \end{array}$ & $\begin{array}{l}\text { Verificar associação entre } \\
\text { anquiloglossia } \\
\text { amamentação. }\end{array}$ & $\begin{array}{l}130 \text { recém- } \\
\text { nascidos }\end{array}$ \\
\hline $\begin{array}{l}\text { Daggumati } \\
\text { et al. } 23 \\
(2019)\end{array}$ & $\begin{array}{l}\text { Caregiver perception of } \\
\text { speech quality in } \\
\text { patients } \\
\text { ankyloglossia: with } \\
\text { Comparison between } \\
\text { surgery and non- } \\
\text { treatment. }\end{array}$ & $\begin{array}{lcr}\text { Determinar } & \text { se } & \text { há } \\
\text { diferenças na fala entre } \\
\text { pacientes submetidos a } \\
\text { frenectomia } & \text { e } & \text { não } \\
\text { submetidos } & \text { a } & \text { esse } \\
\text { tratamento. } & & \\
\text { rato }\end{array}$ & $\begin{array}{l}\text { Cuidadores de } \\
67 \text { pacientes }\end{array}$ \\
\hline
\end{tabular}

DISCUSSÃO

A anquiloglossia é uma anomalia genética lingual rara com predileção ao sexo masculino. Essa condição causa alterações nas estruturas e funções do sistema estomatognático, prejudicando o convívio social do indivíduo ${ }^{13}$. O freio lingual é uma estrutura anatômica que tem importante participação no ato da sucção, fala e alimentação. Um freio lingual curto e aderido ao soalho bucal dificulta os movimentos da língua, o que pode prejudicar 
as diversas funções dessa estrutura ${ }^{24}$.

O leite materno é o alimento mais adequado para todo recém-nascido. Para a correta extração do mesmo, a função de sucção do bebê deve estar em equilíbrio. Todavia, ainda não existe uma relação bem esclarecida entre anquiloglossia com 0 aleitamento materno $^{16}$. Os relatos de mães logo nos primeiros dias de vida do bebê sobre a dificuldade de sucção, são bem comuns. Isso pode ser causado pela anquiloglossia que, nesse contexto, se apresenta como um fator de risco para o sucesso da amamentação $0^{22}$.

Para o correto diagnóstico de alterações que acometem o freio lingual, deve-se obter adequada visualização. Porém, quanto mais posteriorizado e submucoso o freio é, mais dificuldade 0 avaliador terá de examinar a região ${ }^{25}$. Na maior parte dos casos é possível a visualização do frênulo lingual a partir da manobra de elevação das laterais da língua. Quando o freio se encontra recoberto por uma cortina de mucosa deve-se utilizar uma manobra simultânea de elevação das margens laterais e posteriorização da língua ${ }^{26}$.

A presença de anquiloglossia em crianças e adolescentes pode levar a vários problemas que afetam a saúde e o bem-estar destas. Tais problemas podem envolver dificuldade na amamentação, distúrbios da fala, higiene bucal deficiente e bullying por parte dos pares durante a infância e adolescência ${ }^{21}$. O estabelecimento de uma avaliação multidisciplinar se faz muito importante para crianças que precisam do procedimento de frenotomia $^{18}$. Este pode melhorar a amamentação das crianças ${ }^{15}$.

Muldoon et al. ${ }^{17}$ afirmam em seu estudo que a frenotomia afeta positivamente a amamentação, melhorando o vínculo das mães com os lactentes. Apesar das pesquisas ainda serem escassas, a maioria dos estudos aponta para uma melhora também do desconforto sentido pelas mães após a realização da frenotomia ${ }^{27}$.

Com relação aos métodos de diagnóstico na literatura fica bem esclarecido que o conhecimento de gestantes sobre 0 "Teste da Linguinha" (instrumento de diagnóstico oficial vigente no Brasil) é superficial, especialmente entre as de menor escolaridade e renda. Tal fato reforça a necessidade de estratégias públicas com foco em melhorar a qualidade da assistência oferecida ao binômio mãe-filho ${ }^{20}$.

Há um consenso entre os autores acerca dos efeitos negativos das alterações anatômicofuncionais do frênulo lingual sobre 0 crescimento e o desenvolvimento craniofacial, ainda que a opinião sobre a intervenção cirúrgica precoce não seja unânime ${ }^{28}$.

CONCLUSÃO

A anquiloglossia é uma anomalia que pode ser facilmente diagnosticada precocemente, melhorando a qualidade de vida do bebê e da mãe. A literatura concorda que existe uma relação, mesmo que frágil, sobre o freio lingual e sua implicação na nutrição nutritiva do lactente. Porém, são necessários mais parâmetros e estudos longitudinais para obter dados concisos acerca do correto diagnóstico e momento de tratamento para esses lactentes.

REFERÊNCIAS

1. Brito SF, Marchesan IQ, Bosco CM, Carrilho ACA, Rehder MI. Frênulo lingual: classificação e conduta segundo ótica fonoaudiológica, odontológica e otorrinolaringológica. Rev Cefac. 2008;10(3):343-51.

2. Almeida KR, Leal TP, Kubo $H$, Castro TES, Ortolani CLF. Lingual frenotomy in a newborn, from diagnosis to surgery: a case report. Rev Cefac. 2018;20(2):258-62.

3. Nanci A. Ten Cate, Histologia oral: desenvolvimento, estrutura e função. Rio de Janeiro: Elsevier; 2008.

4. McClellan HL, Kent JC, Hepworth AR, Hartmann PE, Geddes DT. Persistent Nipple Pain in Breastfeeding Mothers Associated with Abnormal Infant Tongue Movement. Int J Environ Res Public Health. 2015;12(9):10833845.

5. Zahra FB, Fawzi R. Manejo de língua em crianças: relato de caso. Pediatr Res. 2016;3(4):249-56.

6. Tunes FA, Giffoni TCR, Goya S, Franzin LCS. Frenotomia lingual em paciente com paralisia cerebral: relato de caso. Rev Uningá. 2017; 51(2):27-31.

7. Martinelli RLC, Marchesan IQ, Berretin-Felix G. Estudo longitudinal das características anatômicas do frênulo lingual comparado com afirmações da literatura. Rev Cefac. 2014; 16(4):1202-7.

8. Silva PI, Vilela JER, Rank RCLC, Rank MS. Frenectomia lingual em bebê: relato de caso. Rev Bahiana Odonto. 2016;7(3):220-27.

9. Lima CB, Maranhão VF, Botelho KVG, Santos Junior VE. Avaliação da anquiloglossia em neonatos por meio do teste da linguinha: um estudo de prevalência. RFO UPF. 2017;22(3): 294-97.

10. Martinelli RLC, Marchesan IQ, Berretin-Felix G. Cartilha do Teste da Linguinha: para mamar, falar e viver melhor. São José dos Campos: Pulso Editorial; 2014. 
11. Botelho LLR, Cunha CCA, Macedo M. O método da revisão integrativa nos estudos organizacionais. Gestão e Sociedade. 2011; 5(11):121-136.

12. Mendes KDS, Silveira RCCP, Galvão CM. Revisão integrativa: método de pesquisa para a incorporação de evidências na saúde e na enfermagem. Texto contexto Enferm. 2008; 17(4):758-764.

13. Morisso MF, Berwig LC, Silva AMT. Anquiloglossia: ocorrência de alterações do sistema estomatognático. RGO. 2012;60(2): 203-8.

14. Pransky SM, Lago D, Hong P. Breastfeeding difficulties and oral cavity anomalies: The influence of posterior ankyloglossia and upperlip ties. Int J Pediatr Otorhinolaryngol. 2015;79(10):1714-17.

15. Wakhanrittee J, Khorana J, Kiatipunsodsai S. The outcomes of a frenulotomy on breastfeeding infants followed up for 3 months at Thammasat University Hospital. Pediatr Surg Int. 2016;32(10):945-52.

16. Fujinaga Cl, Chaves JC, Karkow IK, Klossowski $\mathrm{DG}$, Silva FR, Rodrigues $\mathrm{AH}$. Frênulo lingual e aleitamento materno: estudo descritivo. Audiol Commun Res. 2017;22:1762.

17. Muldoon K, Gallagher L, McGuinness D, Smith V. Effect of frenotomy on breastfeeding variables in infants with ankyloglossia (tonguetie): a prospective before and after cohort study. BMC Pregnancy Childbirth. 2017;17(1):373.

18. Dixon B, Gray J, Elliot N, Shand B, Lynn A. A multifaceted programme to reduce the rate of tongue-tie release surgery in newborn infants: Observational study. Int J Pediatr Otorhinolaryngol. 2018;113:156-63.

19. Martinelli RLC, Marchesan IQ, Berretin-Felix G. Posterior lingual frenulum in infants: occurrence and maneuver for visual inspection. Rev Cefac. 2018;20(4):478-83.

20. Pomini MC, Bordin D, Martins PRD, Demogalski JT, Fadel CB, Alves FBT. Conhecimento de gestantes sobre o teste da linguinha em neonatos. Rev Odontol UNESP. 2019;47(6):341-47.

21. Araujo MDCM, Freitas RL, Lima MGS, Kozmhinsky VMDR, Guerra CA, Lima GMS, et al. Evaluation of the lingual frenulum in newborns using two protocols and its association with breastfeeding. J Pediatr (Rio J). 2020;96(3):379-85.

22. Campanha SMA, Martinelli RLC, Palhares DB. Association between ankyloglossia and breastfeeding. Codas. 2019;31(1):e20170264.

23. Daggumati S, Cohn JE, Brennan MJ, Evarts M, McKinnon BJ, Terk AR. Caregiver perception of speech quality in patients with ankyloglossia: Comparison between surgery and nontreatment. Int J Pediatr Otorhinolaryngol. 2019; 119:70-4.
24. Melo NSFO, Lima AAS, Fernandes A, Silva RPGVC. Anquiloglossia: relato de caso. RSBO. 2011;8(1):102-7.

25. Sanders I, Mu L. A three-dimensional atlas of human tongue muscles. Anat Rec (Hoboken). 2013;296(7):1102-14.

26. Martinelli RLC. Validação do protocolo de avaliação do frênulo da língua em bebês [tese]. Bauru: Faculdade de Odontologia de Bauru, Universidade de São Paulo; 2016.

27. Procopio IMS, Costa VPP, Lia EN. Frenotomia lingual em lactentes. RFO UPF. 2017;22(1): 114-19.

28. Pompeia LE, llinsky RS, Ortolani CLF, Faltin Júnior $\mathrm{K}$. A influência da anquiloglossia no crescimento e desenvolvimento do sistema estomatognático. Rev Paul Pediatr. 2017;35(2): 216-21.

\section{CONFLITO DE INTERESSES}

Os autores declaram não haver conflitos de interesse

AUTOR PARA CORRESPONDÊNCIA

Juliana de Godoy Bezerra Medrado

Universidade de Pernambuco - UPE

Rua Cícero Monteiro de Melo, s/n São Cristóvão, 56503-146 Arcoverde-PE, Brasil

Telefone: (87)992079537

E-mail: juliana.godoy@upe.br

Submetido em 11/05/2020

Aceito em 23/10/2020 